\title{
L'éducation pour la santé durable comme levier de transformation sociale
}

\section{Education for sustainable health as a lever for social transformation}

\author{
Jacqueline Descarpentries ${ }^{1}$ \\ ${ }^{1}$ Laboratoire EXPERICE, Paris 8 - Vincennes, Saint-Denis, France, jacqueline.descarpentries@wanadoo.fr
}

RÉSUMÉ. Cet article repose sur une thèse centrale forte : l'usage social et politique de la recherche en santé publique mène au constat qu'il ne peut pas exister de dispositifs d'éducation à la santé favorables au développement durable, sans une justice cognitive dans la mesure où, l'épistémologie est la première source des inégalités que ce soit dans les domaines de l'éducation, ou de la santé, ou du développement durable. Pour préciser cette thèse, l'article est une première étape d'une longue réflexion qui tente de répondre à la question suivante : En quoi, jusqu'où et comment les épistémologies du Sud sont-elles une grille de lecture pertinence pour la construction d'une éducation pour la santé durable ? Pour amorcer cette démarche, une première réponse est consacrée à un ensemble de questions autour de la critique des épistémologies dominantes euro-centrées du Nord en interrogeant l'épistémé de la recherche en santé publique dès qu'elle fait un usage social et politique de l'éducation dans le champ de la santé publique et du développement durable. Une conclusion ouvre des portes au Buen Vivir et à sa transférabilité dans les recherches pour une éducation pour la santé durable critique.

ABSTRACT. This article is based on a strong central thesis: the social and political use of public health research leads to the observation that there can be no health education devices conducive to sustainable development without cognitive justice to the extent that epistemology is the primary source of inequality in the areas of education, health, or sustainable development. To clarify this thesis, the article is a first step in a long reflection that attempts to answer the following question: How, how far and how the epistemologies of the South are a reading grid relevance for the construction of an education for sustainable health? To begin this process, an initial response is devoted to a series of questions around the critique of dominant euro-centered epistemologies in the North by questioning the episteme of public health research as soon as it makes a social and political use of information, education in the field of public health and sustainable development.

MOTS-CLÉS. Santé publique, doxa, justice cognitive, savoirs scientifiques, savoirs écologiques et savoirs prudents, épistémologie du Sud, épistémicide, extractivisme, buen-vivir.

KEYWORDS. Public health, doxa, cognitive justice, scientific knowledge, ecological knowledge and cautious knowledge, southern epistemology, epistemicide, extractivism, buen-vivir.

\section{Introduction}

J'ai bien conscience qu'en situant mes derniers travaux foucaldiens dans le tournant de la nouvelle philosophie critique, j'introduis des questions autour du matérialisme dans l'analyse des politiques de la vie, alors que Michel Foucault dans son analyse de la biopolitique (1974) a toujours pris soin de ne pas nommer les inégalités (prenant de la distance avec le marxisme), mais de nommer les pouvoirs exercés pour leur construction. $\mathrm{Si}$, à travers mes travaux, j'ai tenté de nommer les conditions qui déterminent un dispositif -au sens foucaldien- de l'éducation à la santé, en large part : les conditions de prévention et d'accès à la santé par l'éducation, j'ai pu préciser comment les savoirs épistémologiques ont un effet de pouvoir sur les politiques publiques à la fois de recherche, et d'intervention éducative et sur les processus de subjectivation des membres d'une société donnée. Or, à l'ère de la santé mondiale («Global Health»), une configuration biopolitique inédite des politiques de la vie émerge où l'éducation à la santé joue un rôle géopolitique en tant que biopouvoir humanitaire ${ }^{1}$ en participant aux interventions sanitaires dans les pays. Les politiques de l'éducation à la santé sont effectivement humanitaires car elles sont devenues la forme par excellence des politiques de la vie ${ }^{2}$. Aussi,

\footnotetext{
${ }^{1}$ http://www.fmsh.fr/fr/college-etudesmondiales/24192

${ }^{2}$ Didier Fassin dans son article de 2006 intitulé Michel Foucault : sociologue ? "Il faut entendre « humanitaire » non pas comme le champ auto-délimité par les agents des grandes organisations non gouvernementales, mais comme une catégorie reposant sur le (c) 2018 ISTE OpenScience - Published by ISTE Ltd. London, UK - openscience.fr 
l'éducation à la santé entrant dans l'ère de la prévention mondiale des maladies dans le cadre des objectifs de développement durable ${ }^{3}$ et de la Charte Mondiale de la santé des populations ${ }^{4}$, dans cette immixtion de l'humanitaire dans la politique de santé publique et de développement durable planétaire, je pars de l'ensemble de mes travaux critiques pour introduire de nouvelles questions sur la pertinence des travaux des Epistémologies du $\mathrm{Sud}^{5}$, comme un tournant de lecture de la critique de la critique. Autrement dit, je tente de réfléchir à des pistes de recherches pour une éducation pour la santé durable, équitable, inclusive et démocratique qui ne peuvent, selon moi, se construire qu'à travers les théories critiques et leur tournant critique, car « la pensée occidentale moderne est abyssale. Elle est un système visible et invisible de distinctions, les distinctions invisibles constituant les fondations des visibles. Les distinctions invisibles sont établies à travers des lignes radicales qui divisent la réalité sociale en deux domaines, le domaine de "ce côté-ci de la réalité sociale" et le domaine de "l'autre côté de la ligne". La division est telle que "l'autre côté de la ligne" disparaît comme réalité, devient non existante, et est produite comme non existante » (De Sousa Santos p. 173).

\section{De la biopolitique euro-centrée à une bio-légitimité des expériences}

Le développement durable d'une éducation pour la santé durable, équitable, inclusive et démocratique est effectivement dépendant de multiples enjeux sociaux et politiques mondiaux tels que le développement économique, tributaire du commerce des matières premières (l'Afrique étant notamment un des principaux réservoirs du monde), des conflits internationaux et locaux (affrontements ethniques...), des puissances économiques et politiques de plus en plus en concurrence ouverte, des politiques financières liées aux pouvoirs locaux, culturels et religieux... A cette complexité s'ajoutent la montée en puissance de phénomènes religieux, la mondialisation des migrations et la prise de conscience environnementale qui obligent à admettre que Nord et Sud se trouvent de fait solidairement responsables de cette économie bio-aspirée (Beynus, J, 1997). De plus, il s'exerce depuis toujours, des savoirs idéologiques qui tendent à démontrer l'asymétrie de domination et la possibilité politique de l'émancipation.

Dans ces temps de conflits, de grandes incertitudes, de remise en cause des existants et des références, ces savoirs sont confrontés à l'arrivée de tous ceux et toutes celles qui de par le monde, du local à l'international, prennent la parole qu'on leur confisquait. En effet, dans ce nouvel ordre mondial, où les effets iatrogènes du néolibéralisme (Laval, 2018) sur la santé, l'éducation et sur l'environnement sont à la fois planétaires, globaux et singuliers, émerge un pouvoir des sujets «subalternes», discriminés, minorisés par la science, l'économie et le politique. Des pouvoirs politiques subis aux pouvoirs d'émancipation, des prises de paroles s'organisent, des actions de résistance se construisent, comme autant d'activités alternatives, pour vivre une vie digne au quotidien, en cherchant à dépasser la simple gestion des calculs sur la vie tels que les promeuvent certaines

principe d'un traitement moral de la vie humaine qui est placée au-dessus des autres valeurs et faisant l'objet de disputes entre les acteurs qui cherchent à s'en approprier les bénéfices symboliques » in revue Sociologie et sociétés, Volume 38, Numéro 2, automne, p. 35-48, 2006.

${ }^{3}$ http://www.thelancet.com/journals/lancet/article/PIIS0140-6736(17)32336-/fulltext?elsca1=tlpr

http://mobile.lemonde.fr/sante/article/2017/09/15/sante-les-trop-lents-progres-vers-les-objectifs-du-developpement-

durable_5186469_1651302.html)

${ }^{4} \mathrm{http}: / /$ www.wfpha.org/charter/the-charter

5 "L'épistémologie du Sud" que j'ai proposé vise la récupération des savoirs et pratiques des groupes sociaux qui, par la voie du capitalisme et du colonialisme, ont été historiquement et sociologiquement mis dans la position de n'être que l'objet ou la matière première des savoirs dominants, considérés comme les seuls valides. Les concepts centraux de l'épistémologie du Sud sont la sociologie des absences, la sociologie des émergences, l'écologie des savoirs, et la traduction interculturelle. Il ne s'agit d'ailleurs vraiment pas d'une épistémologie, mais d'un ensemble d'épistémologies. Au contraire des épistémologies du Nord, les épistémologies $d u$ Sud cherchent à inclure le maximum d'expériences et de connaissances du monde. Ainsi, elles englobent les expériences de connaissance du Nord après les avoir reconfigurées. Ainsi, se construisent des ponts insoupçonnés d'intercommunication, assurant notamment la liaison avec les traditions occidentales qui ont été marginalisées, discréditées ou oubliées du fait de la mise en place du canon de la science moderne au XIXème siècle. » De Sousa Santos B., The World Social Forum and the Global Left, Politics and Society, 36(2), 2008. 
politiques publiques délétères. Partant des pôles d'indignation et à ceux de la dénonciation, des lignes de résistance se sont formées au niveau planétaire par la diffusion mondiale des savoirs structurant de nouvelles technologies de pouvoir, de savoirs de «contre-pouvoirs» à l'expansion de la mondialisation.

Des pouvoirs d'auto-gestion et d'auto-protection pour une vie bonne (le buen-vivir) orientent des conduites possibles vers des champs d'actions possibles (Acosta, 2014), non seulement en contrepouvoirs du développement des sociétés capitalistes inégalitaires, mais aussi en vue de développer une auto santé durable (Andrieu, 2012) et une politique de santé collective. Des expériences de savoirs pour maintenir ou améliorer la santé s'autoorganisent comme forme de contre-pouvoirs aux modèles dominants des politiques publiques de santé «button up » par la systématisation des expériences comme processus de reconstruction collective pour transformer l'action sociale (Gonvales, 2018). En fait, dans ce jeu de savoir et de pouvoir l'éducation dans le champ de la santé se révèle un champ de bataille mettant aux prises une volonté hégémonique des modèles euro-centrés et anglo-saxons de recherche, d'intervention de la santé publique et des modèles émancipateurs concurrents voire contradictoires dont les rapports au corps, à la santé et à l'environnement portent la trace profonde qui se traduisent dans une politique de la vie que désigne le dispositif révélateur de la considération portée aux êtres humains ${ }^{6}$.

Autrement dit, pour mettre en discussion un ensemble de questions autour d'une éducation pour la santé durable, deux positions épistémologiques sont conjuguées ici: la philosophique critique (Foucault, 2013) couplée à la sociologie critique et la sociologie de l'émancipation dans la perspective énoncée dans le tournant des nouvelles théories critiques (Frère, 2015 ; Boltanski, 2009 ; Keucheyan, 2017 ; Honneth, 2015 ; Trom, 2018) et les épistémologies du Sud de De Sousa Santos (2016), en particulier autour de la description des 5 logiques de production des absences et d'une conclusion ouverte sur le buen vivir comme transformation sociale et politique de la santé par l'éducation critique. Toutes les formes d' "épistémologies de surplomb» sont récusées ici pour favoriser une approche «continuiste» qui ne fait pas du ou de la philosophe ou du ou de la sociologue le ou la seul.e détenteur-trice des clefs de l'«émancipation », à l'exclusion des autres agents sociaux considérés comme inconscients devant leur propre position de dominés par l'euro-centrisme des savoirs coloniaux (Goody, 2006, Grosfield, 2010). Aussi, pour tenter de rendre visible l'invisible qui fonde les pratiques d'éducation à la santé, je reprends d'abord « une toute petite histoire des idées euro-centrées » pour comprendre les pratiques discursives de l'éducation à la santé humanitaire de notre présent et pour mieux s'en dégager puisqu'elles sont l'objet de leur propre critique épistémologique des conséquences dans la logique de «production des absences » ( De Sousa de Santos, 2016).

Dans l'analyse du dispositif de la recherche en éducation à la santé rappelons que dès le XIXe siècle, en France, la recherche scientifique se construit autour du positivisme scientifique qui demande de s'en tenir aux faits et aux relations entre les faits pour exprimer des causalités ou des lois de portée générale. Il précise les déterminismes et comment tout peut être connu rationnellement, y compris les comportements humains. La seconde moitié du $\mathrm{XX}^{\mathrm{e}}$ siècle en Europe a vu émerger différentes tentatives d'universalisation des méthodes de recherche. Celles-ci se sont constituées en réponse au monisme méthodologique issu du Cercle de Vienne, stipulant l'unité de la science et la validité du projet d'une science universelle et généralisable, quelles que soient les aires géographiques, les cultures, les populations, et les objets. Puis, dès la fin des années 1960, la philosophie de la biologie euro-centrée a affirmé la spécificité des sciences de la vie et l'obligation de partir des données biologiques de l'épidémiologie pour investir les dispositifs d'éducation à la santé. Au cours des décennies suivantes, on constate l' institutionnalisation quasi-hégémonique de modèles de recherche et d'intervention euro centrés ou nord-américains au cœur des politiques publiques de promotion, de prévention et d'éducation à la santé, qui soutiennent autant la diffusion des savoirs anatomo-centrés

\footnotetext{
${ }^{6}$ Il nous appartient donc de révéler la contradiction entre la valeur supérieure de la vie comme principe et l'inégalité de l'évaluation concrète des vies et penser la bio-légitimité c'est-à-dire la reconnaissance de la vie comme bien suprême. (Fassin, 2006) 
${ }^{7}$ ou de la psyché comme force de production dans les programmes de développement de l'estime de soi, qu'un empowerment émancipateur individuel ou collectif. Dans cette veine, les études épidémiologiques en santé publique précisent les contenus de la nature des maladies, les déterminants de santé dont les facteurs et comportements à risque notamment. Les programmes d'éducation à la santé pathogéniques en émergent dans le but de modifier les comportements par l'éducation à l'autonomie et à la responsabilité. L'usage social et politique des savoirs probants de l'épidémiologie et des modèles d'éducation américano-centrés déterminent ainsi les dispositifs d'éducation à la santé locaux et contingents aux résultats des enquêtes de santé publique. Il détermine aussi l'organisation politique et sociale des programmes de prévention, les publics visés, les contenus didactiques et les pédagogiques. Il détermine ses lieux d'intervention avec ses dispositifs législatifs (l'école, les centres sociaux, l'hôpital....). Ce dispositif d'intervention est en fait basé sur un dispositif scientifique défini par un réductionnisme physico-chimique et sur un idéal positiviste de clarification conceptuelle, qui agit comme une vision absolutiste et dogmatique de la science dont l'usage social et politique participe aux prises de décision dans les politiques publiques de santé nationales, européennes et internationales.

Des normes de savoirs exercent donc un ensemble de pouvoir sur la production et la reproduction sociale de la norme de santé publique ${ }^{8}$ dans les dispositifs d'éducation à la santé français notamment et en grande partie du Nord de l'Europe mais aussi dans les pays anglo-saxons. Ils tendent à se généraliser à travers les programmes d'éducation à la santé de l'UNESCO ${ }^{9}$. L'épidémiologie y joue un rôle majeur en déterminant la norme positiviste de la vie par sa mathématisation ${ }^{10}$ qui exerce un pouvoir de gouvernement de la vie et des corps (Fassin, Memmi, 2004) par un effet de pouvoir des savoirs de la rationalité, déjà largement démontrés par l'Ecole de Frankfort (Horkheimer, 1974) et par l'usage social et politique de l'épistémé de la recherche en santé publique (Foucault, 1966). Une technologie de contrôle des formes de recherche (Foucault, 1969), des méthodes d'intervention et des conceptualisations de recherches scientifiques en éducation à la santé s'imposent donc par une biopolitique (Foucault, 1974) pour le contrôle de la vie, instaurant une politique de la vie du point de vue des épistémologies du Nord.

En effet, au niveau national français, européen et international, la mathématisation de la vie, souvent placée sous le seul angle du logos de l'épistémologie, promue par l'épistémé de la recherche en santé publique, agit comme une forme inédite de biopolitique de la vie au service d'une souveraineté (Foucault, 2001) autant épistémique, thérapeutique qu'éducative, basée, comme ligne de progrès de la science, soit à travers une logique de tri, soit sur une logique d'intervention dogmatique et normative de prévention «button up ». En effet, il existe un usage social et politique de la normativité biologique inscrite dans la dite image dominante du progrès scientifique des positivistes autant critiquée par Popper, Kuhn, Feyerabend, Bloor, Latour, Foucault...que par des postmodernes (Derrida, Deleuze, Gattari...) ${ }^{11}$ Un dispositif d'éducation à la santé en émerge, participant à une psycho-politique (ByungChul, 2014) et à la biopolitique par la normation des corps (Foucault, 2001) utilisés comme forces de production liées à l'écart entre la situation initiale de l'état de santé des individus et la généralisation doxique des cadres théoriques mobilisés euro-centrés ou anglos-saxons et les situations auxquelles ils sont appliqués.

\footnotetext{
${ }^{7}$ L'anatomo-politique est conçue comme l'ensemble des disciplines qui s'exercent sur les corps, qui contraignent et enveloppent les comportements, qui dessinent et déterminent un ordre social des choses.

${ }^{8}$ Voir notamment les normes européennes de santé publiques https://europa.eu/european-union/topics/health_fr

${ }^{9} \mathrm{http}: / /$ unesdoc.unesco.org/images/0024/002464/246453f.pdf

${ }^{10}$ Fassin D., (http://id.erudit.org/iderudit/01637) : Dans sa conférence à l'Université de Bahia intitulée «Les mailles du pouvoir », Michel Foucault y affirme : "La vie est devenue maintenant un objet du pouvoir. Jadis, il n'y avait que des sujets juridiques dont on pouvait retirer les biens, la vie aussi d'ailleurs. Maintenant, il y a des corps et des populations » (1994, p. 194). La vie se dissout donc dans ces deux objets: les corps et les populations. Soit, dans la perspective d'une analyse du pouvoir : la discipline et la régulation, l'anatomo-politique et la biopolitique. La question de la vie elle-même, comme forme et comme enjeu, comme ce qui constitue la matière du vivant et ce qui fait l'expérience des vivants, la vie dans sa signification savante et dans le sens commun semble s'effacer à mesure que l'on entre dans ce qu'il appelle d'abord " pouvoir normalisation », 2007.

${ }^{11}$ Voir la très bonne synthèse de ces critiques épistémologiques dans le dernier ouvrage de Larry Laudan de 2017
} 
Des modèles euro-centrés de la normalité des corps sont effectivement utilisés dont celui de l'homme blanc (Le Blanc, 2007), hétéro-normé, sexué (Ayouch, 2017) et racialisé (Dorlin, 2017) par une essentialisation du genre dans les formes de domination bio-médiales (Dorlin, 2018), mais aussi par une fascination pour une essentialisation de la santé humaine au dépens de l'universel de l'expérience humaine individuelle et sociale de la santé et ce quels que les soient les contextes géopolitiques. On notera aussi qu'un modèle d'éducation américano-centré (empowerment) pour le développement d'une estime de soi est nécessaire au développement du capital mondial (Boltanski, 2009) qui n'est rien d'autres qu'un des fétiches au service du même capitalisme. En occident, pour Zizek (2015), notamment, le développement de soi, la pratique du bouddhisme, les assertions contre « le système » sont autant d'éléments à la mode qui s'opposent en apparence au capitalisme par un arsenal rhétorique qui permet d'organiser le monde de l'éducation à la santé afin de le rendre performatif. En effet, une machine bien réglée produite par une longue culture narrative scientifique avec les mêmes arguments et rhétoriques du Nord au Sud et souvent du Nord vers le Sud, se solde par un faible niveau d'incorporation et de subjectivation des changements de comportements attendus et se réitère à l'infini dans les prises de décisions politiques de prévention par l'éducation à la santé au nom du bien commun et de la justice sociale. A cela s'ajoute la puissance d'expression des prédicateurs de l'Anthropocène (Chateaurayneau, Debaz, 2017) qui serait de s'organiser pour surmonter les controverses et les conflits écologiques. Si pour les uns, il s'agit de nommer la complexité des problèmes de l'éducation liés à la santé humaine et à la nature tout en réfutant tout basculement de l'hégémonie épistémologique pour continuer à modéliser une trajectoire des prises de décisions de prévention ou de promotion de la santé quelles que soient les aires géographiques; pour d'autres, il s'agit d'œuvrer à ouvrir les sciences à la co- production des savoirs.

Ainsi, les pratiques discursives de l'éducation à la santé de notre présent, au niveau national français européen et international, produit de la pensée euro-centrée et anglo-saxonne, induisent des dispositifs normatifs par l'opérativité même des modèles scientifiques privilégiés au service du néolibéralisme (Laval, 2018) où l' universalisme est confondu avec l'uniformité (Julien, 2018), l' universel avec l'ethnocentrisme (Balibar, 2016), où le multiculturalisme est confondu avec le relativisme (Laudan, 2017), où l'écart entre les idées est confondu avec la différence avec l'autre et l'altérité ( Julien 2018), la conceptualité est confondue avec l'empirisme, l'expérience avec les faits épidémiologiques. Les Evidence Base Practices sont généralisées à l'éducation à la santé, quelles que soient les aires géographiques, les enjeux géopolitiques et géostratégiques, soutenues par les financements des laboratoires de santé publique, par des laboratoires pharmaceutiques et par les organisations internationales. Une confusion reste entretenue dans les politiques de promotion de la santé souvent centrées sur les politiques curatives et bien moins sur la prévention et la préservation de l'environnement au nom de l'universalité du droit à la santé pour tous et toutes qui serait une garantie d'une politique publique éthique, équitable,durable, sans tenir compte des savoirs écologiques, ni même la sociologie des absences créée par l'hégémonie des savoirs doxiques de la santé publique eurocentrée et anglo-saxonne. Modèle de recherche entretenue et valorisé y compris dans l'empirisme du développement durable avec ses enjeux politiques au sein des organisations internationales notamment, et des partis politiques pour l'écologie. Il faut bien reconnaitre que ces modèles ont des effets performatifs sur les esprits. Nous n'avons plus un «anthropos » indifférencié, mais plutôt des « systèmes mondes » qui organisent les rapports au savoir et au pouvoir qui invisibilisent les formes de gouvernement fondées sur des asymétries profondes qui organisant de manière très inégale les flux des savoirs, des pouvoirs, des matières, des énergies, des marchandises et des capitaux à l'échelle globale (Albe, Popa, Berdah. 2018). Au lieu d'accepter de travailler sur les écarts entre les épistémologies, en dehors des aires géographiques, des tensions subsistent entre les chercheur.es des savoirs probants quantitatifs des épistémologies du Nord et la systématisation des expériences pour transformer l'action sociale avec les populations des villes, des campagnes, des forêts et des eaux et avec des chercheur.es des épistémologies du Sud. Le monde abyssal entre les deux postures entretient autant des pouvoirs individuels, collectifs et institutionnels. Pour les uns, le scénario pratico réaliste a ses vertus. Il s'élabore autour de l'idée que le monde ne va pas si mal et que les humains de la planète 
vont se soumettre aux injonctions comportementales fondées sur de vieux modèles du XVIII siècle de l'hygiénisme, qui alimentent les mêmes discours sur la prévention aujourd'hui (Parayre, 2018). Pour les autres (les alternatifs-optimistes), il faut tendre vers des modèles coopératifs, d'économie alternative, avec de «vraies » valeurs humanistes, avec beaucoup d'intelligence collective, en mettant en avant l'intelligence pratique des personnes « ordinaires » (Fressoz, 2012).

Or, nous ne pouvons que faire le constat d'un usage social et politique des savoirs probants dans les prises de décision en matière de politiques publiques de santé qui semblent être la seule garantie de la scientificité et la reproductibilité d'un ensemble de connaissances euro-centrées et anglo-saxonne autant dans le Nord que sur le Sud. C'est-à-dire qu'une hégémonie impérialiste ancienne des savoirs, piège d'une souveraineté des épistémologies du Nord qui soutient un usage social et politique du positivisme malthusien, gère le gouvernement de la vie et du corps de l'autre passif à normer, à dresser ou à redresser par un modèle d'éducation universalisable, transférable, plus coercitif qu'émancipateur. L'éducation est effectivement transformée en outil d'intervention de la santé publique au même titre que le dépistage, la vaccination ${ }^{12}$. Or, si l'épidémiologie reste un outil d'aide à la décision des politiques publiques de santé en quoi, jusqu'où et comment son épistémologie doit -elle déterminer les modèles de recherches, de l'éducation et les modalités d'intervention sur les populations par ces approches universalisées, populations d'ici et de là-bas, populations du Nord et population du Sud ? Si elle nomme les déterminants de santé, pourquoi son épistémologie devrait-elle déterminer des liens de causalités entre la transmission des savoirs sur ces mêmes déterminants et la modification des comportements ?Pourquoi ces épistémologies supportent l'idée de travailler sur les populations et non pas avec les populations qui vivent sur des territoires ? En fait, la mathématisation de la vie sert de modèle hégémonique des politiques publiques de santé qui sert d'alibi pour contourner les souverainetés nationales garanties aux populations du Sud (Nugyen, 2017) ${ }^{13}$.

Autrement dit, les recherches en éducation à la santé du Nord soutiennent l'idée d'un relativisme conséquent au niveau national européen et international. C'est-à-dire que la science est aussi une entreprise intégralement sociale et politique où s'exercent des hégémonies méthodologiques, de conceptualisation et de méthodes d'intervention depuis longtemps reconnues (Gramsci, 1983). Ces politiques de recherches participent activement, non seulement à une spécificité des formes de domination de la prévention par la science biomédicale et ses modes de financements, qui ont cours dans certains contextes des politiques publiques de santé du Nord. Elles posent aussi tout un ensemble de questions sur la possibilité d'une résistance à la réalité normative établie qui semblait verrouillée pour les anciens auteurs critiques notamment, à travers la conclusion adornienne pessimiste de l'immixtion des forces capitalistes dans la formation même de la subjectivité, et donc au plus « intime» du corps du sujet. Or, le tournant des nouvelles théories critiques (Frère, 2015) et les Epistémologies du Sud, comme mouvement des citoyens ( De Sousa Santos, 2016) apportent une conception de l'acteur social conscient et critique de la domination qu'il subit et échappe ainsi à une idéologie mystificatrice (Keucheyan, 2017 Boltanski 2009, Thévenot, 1991, Fraser 2011, Honneth, 1992). Le sujet social adopte en réalité une posture critique, dont témoigne le regain contemporain des mouvements sociaux portés par un universel rebelle (Julien, 2018). C'est ainsi que des modèles d'éducation à la conscientisation et à la libération ré-émergent du Sud (Hooks, 2018) pour repenser les pratiques d'éducation à la santé. La critique de l'hégémonie des modèles nord-américains de l'éducation utile au développement du capitalise se dispense dans les milieux de la recherche en sciences humaines et sociales. Une volonté de libération des formes d'aliénation et d'oppression s'organise, l'émancipation des groupes sociaux marginalisés se manifeste et organisent des territoires en résistances (Granjon, 2019)

\footnotetext{
${ }^{12} \mathrm{La}$ « biopolitique », en tant qu'elle est - littéralement - une politique de la vie, c'est-à-dire une politique qui a le vivant pour objet et les vivants pour sujets, c'est une politique des populations, celle qui mesure et régule, construit et produit des collectivités humaines à travers des taux de mortalité et des programmes de planification familiale, à travers des règles d'hygiène et des contrôles des flux migratoires.

${ }^{13}$ https://www.canal-.tv/video/fmsh/entretien_avec_vinh_kim_nguyen.31411

(c) 2018 ISTE OpenScience - Published by ISTE Ltd. London, UK - openscience.fr 


\section{Colonialité des curricula: un système invisible, épistémicide et extractiviste}

$\mathrm{Au}$ demeurant, force est alors de constater que ce système invisible des savoirs s'est effectivement imposé aux praticiens, praticiennes de l'éducation à la santé par l'usage doxique social et politique de la recherche en épidémiologie, qui agit aussi au niveau de l'usage social et politique des curricula didactiques de l'éducation à la santé autant dans les aires géographiques du Nord et du Sud le plus souvent. Ceux-ci s'avèrent épistémicides et extractivites, dès lors que l'épistémologie du Nord impose des méthodes de recherches ou d'interventions éducatives, telles qu'elles sont utilisées du point de vue colonisateur pourrait-on dire (Touchelay, 2017), et ce, quelles que soient les aires géographiques. Effectivement, pour certains auteurs comme Quijano, Escobar, Dussel, Mignolo, Castro, Walsh, Grosfoguel, Cusicanqui, rappellent les formes de colonialité des savoirs qui se dessinent à travers l'hégémonie de la pensée euro-centrée et de l'histoire coloniale ${ }^{14}$.

L'analyse du pouvoir chez Foucault, apporte ici un éclairage intéressant à propos de ces postures quand il précise qu'il s'agit d' "extraire historiquement et empiriquement les rapports de pouvoir, les opérateurs de domination», «au lieu de faire dériver les pouvoirs de la souveraineté » (Foucault 1997: 38) et «de faire ressortir les rapports de domination et de les laisser valoir dans leur multiplicité, dans leur différence, dans leur spécificité ou dans leur réversibilité. Il précise par ailleurs qu'il convienne de «ne pas chercher, par conséquent, une sorte de souveraineté source des pouvoirs ; au contraire, montrer comment les différents opérateurs de domination s'appuient les uns aux autres, dans un certain nombre de cas se renforcent et convergent, dans d'autres cas, se nient ou tendent à s'annuler » (Foucault 1997, p 39).

Autrement dit, c'est moins l'exercice du savoir sur le pouvoir qui est examiné à travers l'exercice de la colonialité des savoirs, mais bien plus des liens de causalité entre le colonialisme et les savoirs incorporés, voire l'exercice de la libération des pouvoirs par la conscientisation de ces problèmes de colonialité qui de fait continue à réduire l'entière responsabilité des pratiques doxiques de recherche et d'intervention éducative en réponse aux problèmes de santé publique aux ex- pays colonisateurs, à des faits extérieurs, et minorer les propres problèmes politiques internes des ex-pays colonisés ? Ces formes de pensées dé-coloniales ne nourrissent-elles en fait pas une forme d'hostilité fétichiste au capitalisme et aux pouvoirs des anciennes colonies au lieu de déplacer les énergies de lutte vers des réponses alternatives endogènes aux problèmes locaux ? La colonialité essentialise l'Occident en un bloc unique et homogène en faisant de l'histoire la seule coupable et responsable de la colonisation. Aussi, je m'interroge sur les processus de subjectivation liée à la colonialité de/ sur l'acteur-trice social.e, réduit.e à ses seules capacités de soumission à l'ordre social du colon et aux politiques de la colonisation des savoirs, des idées, du corps et des comportements de soumission (Balandier, 1982). Dans le fonds, quel est l'usage social et politique de cette colonialité, sachant que je ne nie évidemment pas toutes formes de colonisation qui ont pu s'exercer ou s'exercent encore ? N'existe-t-il pas, en fait, un désir de certains intellectuel.es d'exercer un pouvoir sur les masses et aux étudiant.es d'être plus critique que la critique, lorsqu'ils ou elles se focalisent sur la lutte des classes, intégrée à une philosophie dialectique historique, en créant une lutte des races et des religions, qui selon moi mettent

${ }^{14}$ "le colonialisme dénote une relation politique et économique, dans laquelle la souveraineté d'un peuple est au main d'un autre peuple ou nation, ce qui constitue cette nation comme un empire. A la différence de cela, la colonialité se réfère à un modèle de pouvoir qui a émergé comme conséquence du colonialisme moderne, mais au lieu d'être limité à une relation formelle de pouvoir entre deux peuples ou nations, il est en relation avec des domaines comme le travail, la connaissance, l'autorité et les relations intersubjectives qui s'articulent entre elles à travers le marché capitaliste mondial et de l'idée de race....( il s'exerce) une colonialité des savoirs (qui) survie au colonialisme...»( Maldonado-Torres ,2010). Pour Walsh (2008) « elle se maintient vive dans des textes didactiques, dans les critères d'évaluation d'un bon travail universitaire, dans la culture, dans le sens commun, dans l'image de soi des peuples, dans les aspirations des sujets et dans beaucoup d'autres aspects de notre expérience moderne ». 
en danger les inégalités socio-économiques contre le racisme et le sexisme. Ainsi, si la colonialité des savoirs euro-centrés en éducation à la santé exerçe des pouvoirs racialisés et sexués selon certain.es auteur.es, elle reste une des questions au cœur des tensions entre des chercheur.es dont les controverses s'expriment peu, liées au poids des routines académiques, à la normalisation managériale, aux contraintes de la concurrence pour des postes et des ressources rares qui refroidissent les audaces théoriques dans les prises de position intellectuelle. Ne pourrait-on pas aussi s'interroger sur les inégalités de santé issues des savoirs de la colonialité, sans réduire le problème de la colonisation des idées à la binaire question racialiste, car l'humanité n'a jamais été aussi morbide dans sa redistribution: les riches se soignent mieux, mangent à leur guise, ont accès aux meilleurs soins (souvent dans les pays du Nord) au péril des plus pauvres.

Par ailleurs, je m'interroge sur le pouvoir des langues des colonisés qui sont considérées comme non scientifiques, car inscrits dans des langues non européennes, ou anglo-saxonnes de la recherche, voire non inscrites dans le registre de la vérité des Evidences Bases Practices. Comment alors construire un contre-pouvoir à ces savoirs euro-centrés rationalistes et issus d'une philosophie classique quand Mignolo (2010) affirme que «ces processus, marqués par une violence épistémique, conduisent également à une géopolitique linguistique, car les langues coloniales ou impériales, chronologiquement identifiées au grec et au latin de l'Antiquité, l'italien, le portugais, l'espagnol, le français, l'anglais et l'allemand dans la modernité, établissent un monopole linguistique, méprisant les langues natives et avec comme conséquence, de subvertir les idées, les imaginaires et les cosmogonies des natifs hors de l'Europe ».

Quel est donc l'usage social et politique de ces tensions épistémologiques dans la généralisation des modèles de prévention quelles que soient les populations qui maintiennent la généralisation des modèles de recherche et d'intervention éducative utiles au développement du capital ? Dans le fonds ne vaut-il pas mieux « avoir tort avec Sartre que raison avec Aron»?

En ce sens, faut-il admettre que les programmes d'éducation à la santé accroitraient le pouvoir doxique de normation en opérationnalisant l'importance des connaissances biomédicales euro-centrées liées, non seulement, aux enjeux économiques et politiques néolibéraux du traitement des maladies, avec un discours performatif sur les liens entre la santé et le développement durable équitable, au détriment de la protection réelle de l'environnement et des pratiques de santé de protection pour le bien vivre des populations ?.Ainsi, comment construire une politique de la vie dans laquelle le passage d'une éducation à la santé bio-aspirée standardisée par une biopolitique épistémicide et un biopouvoir ${ }^{15}$ extractiviste, « botton up », iatrogène, destructeur, appauvrissant, à une éducation pour la santé durable bio-«inspirée, circulaire, inclusive, diversifiée, enrichissante et créatrice d'alternatives, éthique, démocratique et transformatrice, dont l'éthique de la recherche est dans l'éthos de la recherche en action elle-même ? Elles sont effectivement épistémicides parce que la recherche des épistémologies du Nord induit un dispositif normatif, hétéro-centrées, genrées et racialisées qui ajoutent des souffrances additionnelles en considérant les populations comme des « vases vides » ou en agissant sur les publics cibles à risques (Black et all, 2017), discriminés, des sujets subalternes, comme les migrants, les indigènes les populations autochtones, les femmes et les minorités en général (ethniques, religieuses, sexuelles quels que soient les pays). Ces publics représentent-ils une menace pour l'expansion capitaliste des industries pharmaceutiques par exemple, quand celles-ci se voient contraintes d'inclure les expériences des malades ou de répondre à des demandes sociales de respect des traditions dans les campagnes de prévention par exemple ? Et, ce même, grâce à des voix de ceux et celles considéré.es comme des minorités qui dénoncent les hégémonies, comme les mouvements des LGBTI par exemple. Or, on le sait, les recherches des épistémologies du Nord continuent à subalterniser, subordonner, marginaliser ou «illégaliser» les expériences de pratiques de protection

\footnotetext{
${ }^{15}$ La biopolitique constitue le biopouvoir, autrement dit le pouvoir sur la vie, fugacement, mais décisivement, théorisé autour de 1976, en particulier dans le dernier chapitre de La volonté de savoir. 
individuelles ou collectives et/ou des groupes sociaux en minorant la valeur suprême de la vie qui est à rappeler dans ces temps de « gestion » des migrations.

Elles sont par ailleurs extractivistes parce que la santé est en lien avec l'environnement et que même si des déclarations politiques décrivent «un resserrement des liens entre l'éducation et la santé, et des engagements internationaux exprimés dans la Charte d'Ottawa pour la promotion de la santé de 1986, le Cadre d'action du Forum mondial sur l'éducation de Dakar de 2000 et, plus récemment, la Déclaration d'Incheon de 2015, Éducation $2030{ }^{16}$, des dispositifs performatifs sont annoncés favorables au lien entre l'éducation à la santé et le développement durable. On parle de santé environnementale (environmental health) qui comprend les aspects de la santé humaine, y compris la qualité de la vie, qui sont déterminés par les facteurs physiques, chimiques, biologiques, sociaux, psychosociaux et esthétiques de notre environnement. Or, une éducation pour la santé inclusive et équitable de qualité dans le respect de l'environnement pour un apprentissage tout au long de la vie est entièrement dépendante des formes d'oppressions créées par l'ordre mondial néolibéral autant de la recherche que l'usage social et politique des déterminants de santé. En effet, je parle d'extractivisme parce que ce terme est initialement employé pour désigner l'exploitation commerciale des produits forestiers au Brésil (Emperaire, 1996) qui désignait à l'origine un mode d'accumulation capitalistique structuré avec les empires coloniaux pour un exportation massive de matières premières vers les métropole; il désigne aujourd'hui une situation où l'État prend en charge une partie des orientations économiques - à travers les nationalisations des mines par exemple - en pensant les industries extractives comme une clé pour le développement (Gudynas, 2009 ; Acosta, 2013), comme en Afrique actuellement ${ }^{17}$. Il désigne aussi le pillage des savoirs par les colonisateurs à travers les colonisations, tout au long de l'histoire. Or, dans la mesure où l'extractivisme est souvent identifié dans des régions caractérisées par la pauvreté et les fortes inégalités sociales, qui cohabitent avec des sols et sous-sols riches en matériaux exploitables, en quoi, jusqu'où et comment une éducation à la santé vivable écologiquement, équitable socialement peut-elle apporter une amélioration du bien-être des populations d'une région donnée dans la mesure où l'effet de percolation attendu par l'implantation d'une exploitation (minière, pétrolière, forestière ...) n'a pas lieu ? L'extractivisme cognitif est tout aussi violent pour les populations du Sud et des Suds dès lors qu'on leur impose un modèle de santé publique euro-centré ou anglo-saxon en dehors de tout dialogue interculturel avec différentes langues scientifiques, artistiques, populaires et militantes (Firpo, 2018); rappelant ainsi, combien ce sont les épistémologies des recherches dans les champs de pratiques de la santé qui sont la premières source des inégalités (De Sousa Santos, 2016) ${ }^{18}$; Nunes Arriscado ,2018). Qu'en est -il par exemple de la campagne de nutrition imposant 5 fruits et légumes par jour, pour toutes les populations. A penser aux enfants des familles les plus démunies de notre planète, comment est-il éthiquement et humainement acceptable de transposer ces modèles franco-français de prévention des maladies cardio- vasculaires à la planète ? L'Extractivisme cognitif est tout aussi violent lorsqu'il s'agit de ne saisir les savoirs écologiques sans penser le respect des savoirs en s'emparant des cultures sans veiller ni à une justice sociale, environnementale, sanitaire et éducative.

\section{Les 5 logiques de production des absences}

En fait, ces premières analyses critiques, très provisoirement, permettent de synthétiser le contenu de cette première étape de réflexion en reprenant les 5 logiques de production des absences de la recherche et de la pratique sociale et politique de l'éducation à la santé telle que l'épistémologie du Nord s'est construite par 5 grandes logiques de production de l'absence définie par De Sousa Santos, 2016, p 272 : la monoculture de la rigueur, qui crée les doubles figures du scientifique, et de l'ignorant

\footnotetext{
${ }^{16}$ http://unesdoc.unesco.org/images/0024/002464/246453f.pdf

${ }^{17}$ voir le site https://intellivoire.net
} 
(le médecin et le profane, l'épidémiologiste et les populations cibles ou à risques); la monoculture du temps linéaire avec les doubles figures de l'évolué et du primitif (les modèles euro-centrés de prévention du «blanc » hétéro-normé et genré versus les comportements de protection ancestraux de l'indigène ou du «négro », de l'autochtone ); la monoculture de la naturalisation des connaissances et des hiérarchies avec les doubles figures du supérieur et de l'inferieur qui pose des problèmes réels autour de la liberté, de l'être et de l'histoire de l'individu soumis par une violence épistémique (Walsh, 2008), comme l'occidentalisme des modèles biomédicaux et technico médicaux implantés versus les cultures tribales, animistes ou alternatives de protection ou de soin) ; la monoculture de l'échelle dominante avec la double figure de l'universalisme ou de la mondialisation versus le local et le particulier (l'universalisme des modèles d'éducation versus les savoirs écologies locaux et contingents au culture locale et artisanat des pratiques de protection), et finalement ; la monoculture des critères de production capitaliste et de la logique productiviste, avec les doubles figures du productif et l'improductif (les campagnes de prévention euro-centrées lancées par les industries pharmaceutiques et les campagnes de prévention communautaires ).

\section{Conclusion provisoire}

Face à ce premier constat, l'enjeu est important pour la paix des générations futures. Nous avons collectivement à nous poser la question des tensions théoriques et morales récurrentes dans la politique de la vie par et pour une éducation pour la santé durable humanitaire dans le respect de la vie humaine.

Nous avons la responsabilité collective de penser une alternative épistémique à l'éducation à la santé en travaillant une épistémologie critique propre au champ de l'éducation pour la santé durable, pour une promotrice de santé émancipatrice, qui sorte la réflexion scientifique des murs sclérosants du déterminisme, de la techno-science, du biopouvoir et de la biopolitique néolibérale. Nous avons à construire collectivement une démarche pour comprendre comment elle peut s'inscrire de manière complexe, incertaine, ambiguë au cœur de différents systèmes de valeurs et d'actions, de nos économies morale et politique en matière de santé publique pour un développement durable éthique et responsable. Travailler les liens entre la santé, l'éducation et l'environnement nous invite donc à travailler les politiques de la vie qui, au-delà d'évidentes différences du point de vue des problématiques locales et contingentes, des contextes et des pays participent d'une même configuration morale dans laquelle la vie physique et psychique s'imposent comme valeur supérieure et la raison humanitaire comme idéal éthique. Des pistes sont à explorer en référence aux travaux d'Eduardo Gudynas et Alberto Acosta, deux grands théoriciens du buen vivir. Celui-ci se définit comme une «occasion de construire une autre société basée sur la coexistence des êtres humains avec la nature, dans la diversité et l'harmonie, à partir de la reconnaissance des différentes valeurs culturelles présentes dans chaque pays et dans le monde ». En tant que discours alternatif à celui du développement durable, le buen-vivir résulte d'une combinaison particulière entre certains principes éthiques de l'ancienne culture andine (défendus par les mouvements indigènes) et les contributions contemporaines de certains courants intellectuels critiques. Des travaux sont à construire à partir des savoirs du buen vivir venant d'Amérique Latine et ses liens avec l'éducation qui s'affirme d'ores et déjà comme un discours critique de l'idéologie de la rationalisation et de l'universalisme propres à la modernité. Quelle est la transférabilité de ce modèle en Europe ou à l'international ? En ce sens, le buen vivir apporterait une nouvelle perspective aux défis du développement durable, dernier avatar du discours sur le développement, qui a tenté d'amortir les critiques du développement sur le terrain économique, social et environnemental (Vanhust, Beling, 2013).

Telles sont les questions auxquelles nous avons collectivement des éléments de réponse qui me semblent prolonger la pensée foucaldienne entre zoé et bios pour une politique de la vie éthique. L'ensemble de ces situations rendent indispensable la rupture avec les logiques de concurrence exacerbée, de guerre économique et de chacun pour soi placées au cœur de la marche du monde par le néolibéralisme. 
La réponse à ces défis appelle des logiques de coopérations à tous les niveaux, du plus local jusqu'à l'échelle mondiale pour développer et organiser la mise en commun des projets, des ressources, des énergies, des savoirs. L'intelligence collective, l'agro écologie restent des levier pour répondre aux besoins les plus quotidiens, pour défricher des voies nouvelles de respect de l'environnement et des politiques de santé collective sur les territoires pour se situer à la hauteur des enjeux de certains Etats et des besoins des populations. L'écologie des savoirs représente un de ces leviers pour une éducation pour la santé durable qui, «non seulement permet de dépasser la monoculture du savoir scientifique, mais favorise également l'idée que les savoirs non scientifiques constituent des alternatives au savoir scientifique » (p. 279). Cette écologie des savoirs «n'entend pas accepter le relativisme [...]. Elle cherche à créer une nouvelle sorte de relation, dite pragmatique, entre le savoir scientifique et les autres types de savoir. Le but n'est pas d'attribuer la même validité à chaque type de savoir, mais plutôt de permettre une discussion pragmatique entre des critères valides et alternatifs sans automatiquement disqualifier ce qui ne correspond pas au canon épistémologique de la science moderne » (p. 278). Dans la mesure où l'écologie des savoirs est «constituée de questions constantes et de réponses incomplètes » (p. 300), elle nous invite à un savoir prudent. Seul, un savoir prudent, conscient de son incapacité à saisir la totalité de la complexité humaine, peut favoriser l'émancipation. "La traduction interculturelle est l'alternative à la fois à l'universalisme abstrait qui fonde les théories générales occidentalo-centriques, et à l'idée d'incommensurabilité entre les cultures. [...] elle tente d'identifier les différences et les similitudes, et de développer, lorsque les circonstances s'y prêtent, de nouvelles formes hybrides de compréhension et d'intercommunication culturelles à même de favoriser les échanges et de renforcer les alliances entre des mouvements sociaux qui luttent, dans des contextes culturels différents, contre le capitalisme, le colonialisme et le sexisme, et pour la justice sociale, la dignité et la décence humaine » (pp. 309-310).

\section{Bibliographie}

Acosta, A. (2013) "Extractivism and neoextractivism: two sides of the same curse". Beyond Development, 2013, p. 61

Andrieu, B. (2012) Une auto-santé, vers une médecine réflexive, Paris : Editions Armand Colin

Balibar, E. (2016) Des Universels, Paris, Galilée

Black, G.F, Davies, A., Iskander. D \& Chambers, M. (2018) Reflections on the ethics of participatory visual methods to engage communities in global health research, Global Bioethics, 29:1, 22-38, DOI: 10.1080/11287462.2017.1415722 https://doi.org/10.1080/11287462.2017.1415722

Boltanski, L et Thévenot, L. (1991) De la justification, Paris, Gallimard

Boltanski, L. (2009) De la critique, précis de sociologie de l'émancipation, Paris, Gallimard

Bruckner, P. (2002) Le Sanglot de l'homme blanc, Seuil, 2002,

Bruno Frère (dir.). (2015) Le tournant de la théorie critique, Paris, Desclée de Brouwer

Byung-Chul, H. (2014) Psychopolitique, le néolibéralisme et les nouvelles techniques de pouvoir, Strasbourg, Editions CIRCE

Callon, M. Lascoumes, P, Barthe, Y. (2001) Agir dans un monde incertain. Essai sur la démocratie technique, Paris, Éditions du Seuil

Canguilhem, G. (1968) Études d'histoire et de philosophie des sciences concernant les vivants et la vie, Paris, Vrin.

Chateauraynaud, F,Debaz, J.(2017) Au bord de l'irréversible, Sociologie pragmatique des transformations, Paris, Editions Petra

Dozon, JP. \&Fassin, D. (2001) Critique de la santé publique, Paris, Balland

Elgas. (2015) Un Dieu et des mœurs, Paris, Présence Africaine

Emperaire, L. (1996) La Foret en jeu (the Forest at Stake): L'extractivisme en Amazonie centrale (Extraction in Central Amazonia). IRD Éditions

Fanon, F. (1962) Les damnés de la terre, Paris, Maspéro

Fassin, D \& Memmi, D. (2004) Le gouvernement des corps. Paris : La Découverte

(c) 2018 ISTE OpenScience - Published by ISTE Ltd. London, UK - openscience.fr 
Fassin, D. (2000), «Entre politiques de la vie et politiques du vivant», Anthropologie et sociétés, numéro spécial « Terrains d'avenir », 24 (1), 95-116.

Fassin, D. (2006), «L'humanitaire contre l'État, tout contre», Vacarme, numéro spécial «Politique non gouvernementale », 34, 15-19.

Fernandes de Oliveira, L.Vera- Candau, M. (2010), «Pedagogia decolonial e educação antirracista e intercultural no Brasil », Educ. rev. vol.26 no.1 Belo Horizonte April.

Ferreira da Rocha, D., Firpo Porto, M., Pacheco, T., (2017) Ecological Distribution Conflicts as Forces for Sustainability The map of conflicts related to environmental injustice and health in Brazil in The EJAtlas: Ecological Distribution Conflicts as Forces for Sustainability https://doi.org/10.1007/s11625-017-0494-5

Foucault, M. (1969) L'archéologie du savoir, Paris, Gallimard

Foucault, M. (1975) Surveiller et punir, Paris, Gallimard

Foucault, M. (1988) Les mots et les choses, Une archéologie des sciences humaines. Paris : Gallimard

Foucault, M. (1997) «Il faut défendre la société », Paris : Les Éditions du Seuil/Gallimard

Foucault, M. (2001) «La gouvernementalité », in : ibid., Dits et écrits II, 1976-1988, Paris : Gallimard (Quarto) pp. 635657

Foucault, M (2008) Le gouvernement de soi, Paris, Gallimard

Foucault, M. (2004) Naissance de la biopolitique, Paris, Gallimard

Foucault, M. (2013) Qu'est -ce que la critique ? suivi de La culture de soi, Paris, Vrin

Fraser, N. (2011) Qu'est-ce que la justice sociale ? Reconnaissance et redistribution, Paris, La Découverte

Freire, P. (1974) Pédagogie des opprimés (suivi de « Conscientisation et Révolution »), petite collection Maspero

Goody, J. (2006) Le vol de l'histoire, comment l'Europe a imposé le récit de son passé au reste du monde, Paris, Folio

Grosforfeld, R. (2010) Vers une décolonisation des « uni-versalismes » occidentaux : le «pluri-versalisme décolonial», d'Aimé Césaire aux zapatistes, in Ruptures postcoloniales, La Découverte

Gudynas, E. (2009) «Diez tesis urgentes sobre el nuevo extractivismo : contextos y demandas bajo el progresismo sudamericano actual », in Jürgen Schludt et al., Extractivismo, política y sociedad, Quito, CAAP/Claes, 2009, p. 187225 [pdf].

Gudynas., E et Acosta, A (2011) «El buen vivir o la disolución de la idea del progreso », in M. Rojas (dir.), La medición del progreso y el bienestar : Propuestas desde América Latina, Foro Consultivo Científico y Tecnológico de México, Mexico, 2011, p. 103.

Honneth, A. (1992) La lutte pour la reconnaissance, Paris, Gallimard

Horkheimer, M (1974) Théorie traditionnelle et théorie critique, Paris, Gallimard

Keucheyan, R. (2017) Hémisphère Nord, Une cartographie des nouvelles théories critiques, Paris, La Découverte

Laudan, L. (2017) Science et relativisme. Quelques controverses clefs en philosophie des sciences, Paris, Editions Métrologies.

Laval, C. (2018) Foucault, Bourdieu et la question néolibérale, Paris : La Découverte

Le Blanc, G (2004) Les maladies de l'homme normal, Paris, Editions du Passant

Nicolas- Le Strat, P. (2016) Le travail du commun, Editions du commun

Pries, L. (2016) Soziologie, Schlüsselbegriffe, Herangehensweisen, Perspektive, Weinheim : Editions Beltz, , Juventa

Smith, S. (2003) La négrologie, Pourquoi l'Afrique meure, Pairs Edition Calmann-Levy

Trom, D. (2018) La sociologie critique ou la dépolitisation de la sociologie de la politique », SociologieS [En ligne], Débats, La situation actuelle de la sociologie, mis en ligne le 19 juin 2018.URL: http://journals.openedition.org/sociologies/7505 